Article

\title{
Global, non-parametric, non-iterative optimization of time-averaged quantities under small, time-varying forcing: An application to a thermal convection field
}

\author{
Hideshi Ishida ${ }^{1}$, Shiho Ihara ${ }^{2}$, Chiharu Okema ${ }^{3}$, Shohei Yamada ${ }^{4}$ and Genta Kawahara ${ }^{5}$ \\ 1 Department of Mechanical Engineering, Setsunan University 17-8 Ikeda-Nakamachi, Neyagawa, Osaka \\ 572-8508, Japan; hideshi.ishida@mec.setsunan.ac.jp \\ 2 Department of Mechanical Science and Bioengineering, Graduate School of Engineering Science, Osaka \\ University 1-3 Machikaneyama-cho, Toyonaka, Osaka 560-8531, Japan; Present address: ]All Nippon \\ Airways Co., Ltd., Shiodome-City Center, 1-5-2 Higashi-Shimbashi, Minato-ku, Tokyo 105-7140, Japan \\ 3 Department of Mechanical Science and Bioengineering, Graduate School of Engineering Science, Osaka \\ University 1-3 Machikaneyama-cho, Toyonaka, Osaka 560-8531, Japan; Present address: Daikin Industries, \\ Ltd., Umeda Center Bldg., 2-4-12 Nakazaki-Nishi, Kita-ku, Osaka 530-8323, Japan \\ 4 Department of Mechanical Science and Bioengineering, Graduate School of Engineering Science, Osaka \\ University 1-3 Machikaneyama-cho, Toyonaka, Osaka 560-8531, Japan; Present address: DENSO \\ Corporation, 1-1 Showa-cho, Kariya, Aichi 448-8661, Japan \\ 5 Department of Mechanical Science and Bioengineering, Graduate School of Engineering Science, Osaka \\ University 1-3 Machikaneyama-cho, Toyonaka, Osaka 560-8531, Japan
}

\begin{abstract}
This study demonstrates a global, non-parametric, non-iterative optimization of time-mean value of a kind of index vibrated by time-varying forcing. It is based on the fact that the (steady) forced vibration of non-autonomous ordinary differential equation systems is well approximated by an analytical solution when the amplitude of forcing is sufficiently small and its base state without forcing is stable and steady. It is applied to optimize a time-averaged heat-transfer rate on a two-dimensional thermal convection field in a square cavity with horizontal temperature difference, and the globally optimal way of vibrational forcing, i.e. the globally optimal, spatial distribution of vibrational heat and vorticity sources, is first obtained. The maximized vibrational thermal convection corresponds well to the state of internal gravity wave resonance. In contrast, the minimized thermal convection is weak, keeping the boundary layers on both sidewalls thick.
\end{abstract}

Keywords: Global, non-parametric, non-iterative optimization; Time-mean quantities; Small time-varying forcing; Ordinary differential equation system (ODEs); Eigenvalue problem

\section{Introduction}

Numerous studies have been carried out in many fields on maxima or minima of time-averaged quantities [1-20]. They include the optimization of temperatures [1-3], heating rate [4], beam to wave power transfer efficiency [5], current density [6], reaction rate [7], flow vorticity [8] and velocity [9,10], drag reductions [11,12], heat transfer rates [13-17], shear stresses [18-20] and so on. However, most of them are parametric optimization, useful in cases where the number of optimization variables is small. Like the field optimization [21-23], however, large or infinite number of the variables prevents us from utilizing the method.

That is the reason why non-parametric methods are applied to solve the types of optimization. However, very little work is available for optimizing time-averaged quantities because of the difficulty of unsteady optimization [24-30]. Srinath and Mittal [24] and Fang and Li [29] applied a continuous adjoint method to obtain the optimal shape of airfoils for maximizing time-averaged lift coefficient or minimizing time-averaged drag coefficient, in conjunction with iterative methods, such as descent or conjugate gradient methods. Similarly, iterative algorithms were utilized to maximize time-averaged system energy efficiency [25], and to maximize time-averaged transmission rate [28], and so on. 


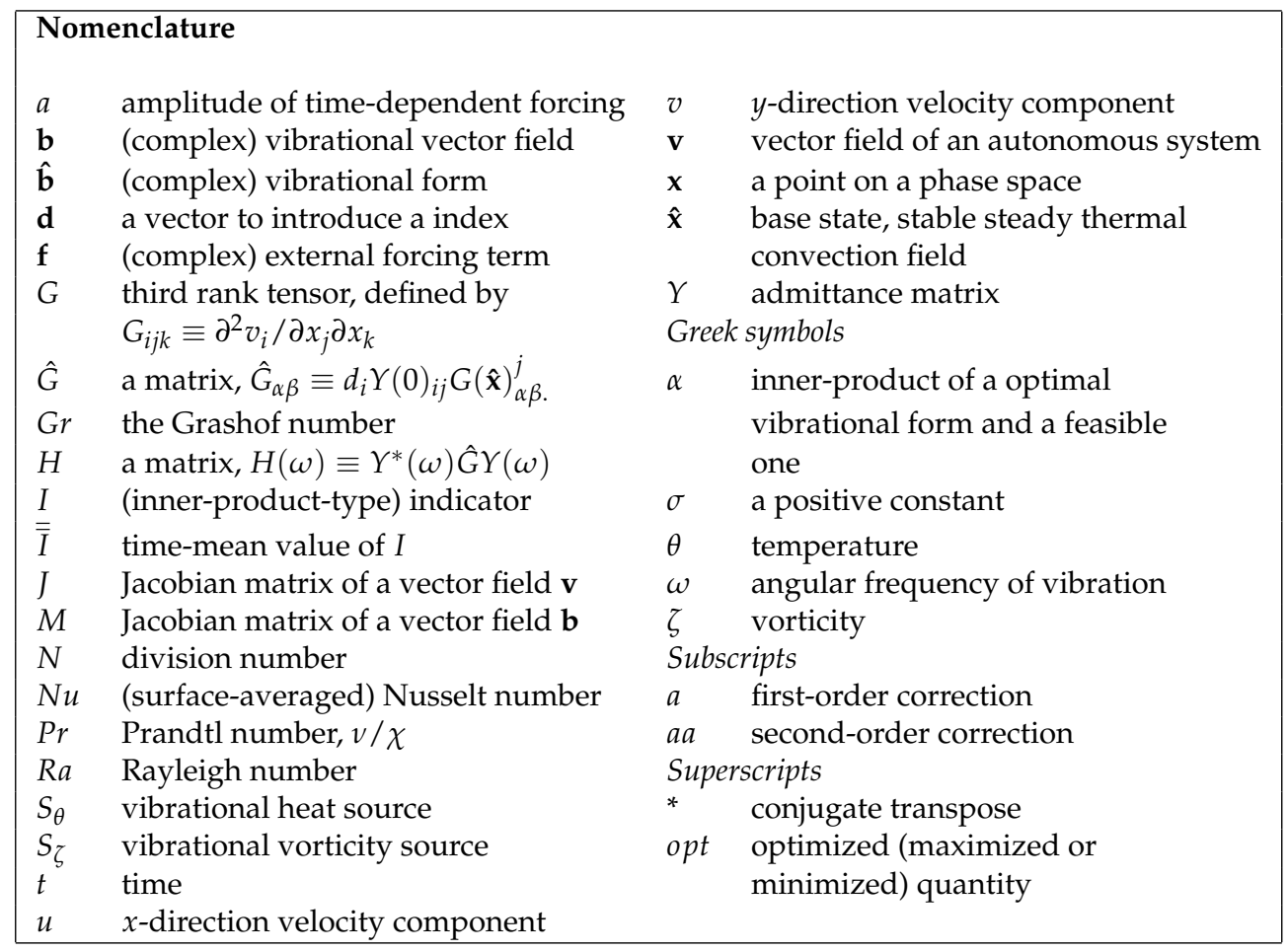

The use of iterative methods is inevitable for the cases governed by nonlinear equations: the optimal solution cannot be obtained by a one-time numerical computation. Accordingly, the application of non-parametric and non-iterative optimizations of time-averaged quantities are limited to special cases to which analytical approaches are useful [31,32]. Nelson et al. [31] proposed the active control of low-frequency random sound in enclosures and, as an example, it is applied to one-dimensional enclosure to minimize time-averaged acoustic potential with the aid of an analytically obtained auto-correlation function. Chen and Muga [32] studied a time-dependent harmonic oscillator and found that the time-averaged energy realized by the optimal, time-dependent engineered scaling function is indeed minimum, reaching its lower bound obtained analytically. In general, however, even if such an optimal solution is obtained, we do not know whether or not the solution is globally optimal. We more or less confront these issues to optimize over a field for maximizing or minimizing a time-averaged quantity.

In this study, a non-parametric, non-iterative method is proposed for globally optimizing a kind of time-averaged quantities vibrated by time-varying forcing. In this method, we shall treat weakly nonlinear response to finite-amplitude vibrational forcing, applying a perturbation theory to a general form of ordinary differential equation system (ODEs) with the amplitude of external forcing taken as a perturbation parameter $[33,34]$. The correction terms are obtained analytically, allowing us to predict the response to arbitrary external forcing on the same base field given by a non-vibrational condition. As long as the base field is stable, steady state, the perturbation expansion is possible, and its second-order truncated expansion [34] provides us with the above-mentioned optimization method. Discretization of governing equations makes it possible for us to apply the method to many practical systems with dumping mechanisms and holonomic constraints, and to obtain the globally optimal vibrational field without any parametric, iterative computations.

As an application of the field-optimization method, we are to address a vibrational thermal convection [35-42] with a horizontal temperature difference in a square cavity, and time-mean heat transfer rate, i.e. Nusselt's number, is maximized and minimized. Recently, Pourgholam et al. [17] found that the time-averaged heat transfer rate on the heated top and bottom walls in a parallel flow channel reaches maximum by a periodically moving blade near the inlet port. Velazquez, Arias and Mendez [16] showed that the maximum time-averaged Nusselt number over the downstream 
portion of a backward facing step is $55 \%$ larger than the value of the steady state when velocity modulation is exerted in the inlet port at the resonant frequency. Such a heat transfer maximization induced by external vibrations is rather intensely examined in natural convections [36-42]. For instance, Kim, Kim and Choi [40] conducted a numerical study to investigate the boundary layer instability in a square cavity with side-wall temperature modulation, and clarified that the maximum time-averaged heat transfer rate is obtained for the oscillation in tune with the frequency of boundary layer instability. They are parametric optimizations with smaller numbers of optimization variables and, to our best knowledge, the spatial distribution of vibrational heat and vorticity sources to optimize the time-averaged Nusselt number is first obtained in this study. The maximization and minimization are simultaneously accomplished by solving an eigenvalue problem of a Hermitian matrix.

We have already proposed a non-parametric, non-iterative method to maximize or minimize total amplitude of vibrational thermal convection, based on the first-order truncated perturbation expansion, and found three intrinsic vibrational modes including the mode of internal gravity wave (IGW) [33]. The optimization presented here is an extension based on the second-order truncated expansion: the expansion includes the correction for nonlinear vibrational effects, making it possible for us to first optimize the time-averaged quantities affected by the nonlinearity.

\section{Methods and Definitions}

\subsection{Approximation of forced oscillation on ODEs}

Considering that a partial differential equation system with holonomic constraints is discretized to be an ordinary differential equation system (ODEs), it is meaningful for us to begin with the following system with forcing

$$
\frac{d \mathbf{x}}{d t}=\mathbf{v}(\mathbf{x})+a \mathbf{f}(\mathbf{x}, t),
$$

where $\mathbf{x}$ denotes a column vector of $N$ components indicating a point on a phase space, $\mathbf{f}$ forcing normalized by the amplitude $a$, and $\mathbf{v}$ vector field without forcing. We assume that it has a stable steady solution (base field) $\hat{\mathbf{x}}$ without forcing and that the amplitude $a$ is sufficiently small. Then taking $a$ to be a perturbation parameter, we are to solve the equation around the steady solution of the form

$$
\mathbf{x}=\hat{\mathbf{x}}+a \mathbf{x}_{a}+a^{2} \mathbf{x}_{a a} / 2+\cdots .
$$

We can obtain the corrections such as $\mathbf{x}_{a}$ and $\mathbf{x}_{a a}$ analytically. The procedure is detailed in Ref. [34]. In what follows, we are to restrict the vibrational forcing $f$ into a single-frequency vibration of the form

$$
\mathbf{f}=e^{i \omega t} \mathbf{b}(\mathbf{x})+\text { c.c., }
$$

where $\omega$ denotes angular frequency of vibration, $\mathbf{b}$ a complex vibrational vector field, and c.c. the complex conjugate terms. Then the steady responses of the first- and second-order corrections are given by

$$
\begin{aligned}
\mathbf{x}_{a} & =e^{i \omega t} Y(\omega) \hat{\mathbf{b}}+\text { c.c. } \\
\mathbf{x}_{a a} & =e^{2 i \omega t} Y(2 \omega)\left(\hat{\mathbf{b}}^{T} Y^{T}(\omega) G(\hat{\mathbf{x}})+2 M(\hat{\mathbf{x}})\right) Y(\omega) \hat{\mathbf{b}} \\
& +Y(0)\left(\hat{\mathbf{b}}^{T} Y^{T}(\omega) G(\hat{\mathbf{x}})+2 M(\hat{\mathbf{x}}) \overline{Y(\omega) \hat{\mathbf{b}}}+\right.\text { c.c. }
\end{aligned}
$$

where $\hat{\mathbf{b}} \equiv \mathbf{b}(\hat{\mathbf{x}})$, and $Y \equiv(i \omega I-J(\hat{x}))^{-1}$, and the overline denotes complex conjugate quantities [34]. A third rank tensor $G$ is defined by $G_{i j k} \equiv \partial^{2} v_{i} / \partial x_{j} \partial x_{k}$, and its associated operation is also defined as $\left\{\mathbf{x}^{T} G \mathbf{y}\right\}_{i} \equiv G_{i j k} x_{j} y_{k}$. I denotes the identity matrix, and $J$ and $M$ denote the Jacobian matrices of $\mathbf{v}$ and b, respectively. 
The tensors $J(\hat{\mathbf{x}}), Y(\omega)$ and $G(\hat{\mathbf{x}})$ are intrinsic properties of a given vector field $\mathbf{v}$ and a base field $\hat{\mathbf{x}}$ without forcing. $Y$ is referred to as an admittance matrix in Ref. [33], corresponding to a frequency response function. In contrast, the complex matrix $M(\hat{x})$ and vector $\hat{\mathbf{b}}$ have the information on the way of vibration, and $\hat{\mathbf{b}}$ is hereafter referred to as vibrational form. This is the optimization variable in this study.

When the expansion (2) is truncated at the first- or second-order, the perturbation expansion becomes the first- or second-order approximation with respect to the vibration amplitude $a$ [34]. Particularly, the second-order approximation has a correction term for the direct-current component caused nonlinearly by a vibrational forcing, making it possible to treat the change in time-mean values by vibration. By modifying two variables $M(\hat{x})$ and $\hat{\mathbf{b}}$, we obtain the response to an arbitrary small-amplitude time-varying forcing.

In order to apply these approximations, the amplitude $a$ must be small enough, mathematically infinitesimal. The applicability of the truncated forms will be examined in Sec. 3.1.

\subsection{Optimization and formalism}

On the first-order approximation, i.e. first-order truncated perturbation expansion, Ishida et al. [33] showed that the maximization of total amplitude, defined by $|Y \mathbf{b}|^{2}$, subject to the normalization condition of vibrational form $\hat{\mathbf{b}}$, is reduced to an eigenvalue problem of a Hermitian matrix $\hat{H}\left(\equiv Y^{*} Y\right)$, where superscripted asterisk ' $*$ ' denotes conjugate transpose. The optimal vibrational form is the eigenvector corresponding to the maximum eigenvalue. This is a non-parametric, non-iterative optimization. In this section, we shall see that a similar formulation is possible to optimize time-mean value of a kind of time-dependent, oscillating quantity on the second-order approximation.

2.2.1. Inner-product type index and the optimization of its time-averaged quantity

The quantity is an inner-product-type index $I$, defined by

$$
I \equiv \mathbf{d}^{T} \mathbf{x}
$$

where $\mathbf{d}$ is a constant real vector. For its time-mean value $\overline{\bar{I}}$ we consider the problem

$$
\begin{array}{cl}
\operatorname{maximize}(\operatorname{minimize}) & \overline{\bar{I}}\left(\hat{\mathbf{b}}, M(\hat{\mathbf{x}}), \partial^{2} b_{i} / \partial x_{j} \partial x_{k}(\hat{\mathbf{x}}), \cdots\right) \\
\text { subject to } & \|\hat{\mathbf{b}}\|^{2} \equiv|\hat{\mathbf{b}}|^{2} / \sigma=1 \\
& M(\hat{\mathbf{x}})=0
\end{array}
$$

where $\sigma$ is a positive constant. As we shall see, the following formulation is unchanged on the condition that the $L_{2}$-norm of $\hat{\mathbf{b}}$ is held fixed. The constant $\sigma$ may be interpreted in such a degree of freedom. The restriction of the vibrational way to the case of $M(\hat{\mathbf{x}})=0$ is for simplification in this study.

On the second-order approximation, $\overline{\bar{I}}$ is a function of $\hat{\mathbf{b}}$ and $M(\hat{\mathbf{x}})$ only, and the problem is reduced to the following eigenvalue problem of a Hermitian matrix $H$ :

$$
H \hat{\mathbf{b}}_{\text {opt }}=\lambda_{\text {opt }} \hat{\mathbf{b}}_{\text {opt }}
$$

where

$$
\begin{aligned}
& H(\omega) \equiv Y^{*}(\omega) \hat{G} Y(\omega), \\
& \hat{G}_{\alpha \beta} \equiv d_{i} Y(0)_{i j} G(\hat{\mathbf{x}})_{j \alpha \beta},
\end{aligned}
$$


and the optimized value of $\overline{\bar{I}}$ can be expressed as

$$
\overline{\bar{I}}_{o p t}=\mathbf{d}^{T} \hat{\mathbf{x}}+a^{2} \sigma \lambda_{o p t}\left\|\hat{\mathbf{b}}_{o p t}\right\|^{2},
$$

where $\lambda_{\text {opt }}$ is the maximum or minimum eigenvalue of the problem (7), and $\hat{\mathbf{b}}_{\text {opt }}$ is its corresponding eigenvector. The second term of RHS of Eq. (8) indicates the increment of time-mean value realized by the optimal vibration. Herein it should be noted that the maximum and minimum values and corresponding vibrational forms are obtained at the same time without any parametric, iterative computations and that they are the globally, not locally, optimal solutions.

Some reasonable constraints of vibrational form can change from the definition of the norm (6) into the form

$$
\|\hat{\mathbf{b}}\|^{2} \equiv \frac{1}{\sigma} \hat{\mathbf{b}}^{*} A \hat{\mathbf{b}}
$$

where $A$ is a positive definite Hermitian matrix. In this case, the optimization problem is reduced to the following generalized eigenvalue problem:

$$
H \hat{\mathbf{b}}_{\text {opt }}=\lambda_{\text {opt }} A \hat{\mathbf{b}}_{\text {opt }}
$$

and the formulation provides again a non-parametric, non-iterative optimization procedure. For simplification, however, we shall not treat such a case of constraint in this study.

\subsection{A sample vector field: $a$ thermal convection}

A base and a vector fields are needed to assess the applicability of the optimization scheme. The sample base field in this study is a two-dimensional, dimensionless thermal convection in a square cavity shown in Fig. 1. A constant temperature difference is given between the left- and right-hand side walls. The top and bottom walls are insulated. The no-slip, zero velocity condition is set on the wall surfaces. The gravity works in the negative $y$ direction.

A phase space is defined by use of vorticity $\zeta$ and temperature $\theta$ defined at inner lattice points within the cavity: when we define $\zeta_{i}$ and $\theta_{i}$ for a natural number $i$, as described in Fig. 2, a sample coordinate $\mathbf{x}$ of a phase space can be defined as

$$
\mathbf{x} \equiv\left(\zeta_{1}, \zeta_{2}, \cdots, \zeta_{L}, \theta_{1}, \theta_{2}, \cdots, \theta_{L}\right)
$$

In this study the division numbers $n$ and $m$ for horizontal and vertical directions, respectively, are equal, i.e. $n=m(\equiv N)$.

The normalized governing equations of a Boussinesq fluid are composed of vorticity and temperature equations, in conjunction with a definition equation for vorticity, which behaves like a holonomic constraint $[33,43]$. In this study these equations are discretized by finite difference method, expressed by the vorticities and temperatures defined on the above-mentioned inner lattice points with the aid of the boundary conditions. Convection and diffusion terms are discretized by QUICK and central difference schemes, respectively. Consequently, we obtain a vector field $\mathbf{v}$ of $\mathbf{x}$.

In this study, the Prandtl number $P r$, the Rayleigh number $R a$, and the Grashof number $G r(\equiv$ $\mathrm{Ra} / \mathrm{Pr}$ ) are fixed at $0.71,10^{5}$ and $1.4 \times 10^{5}$, respectively, so that non-vibrational field evolves to reach a stable steady state of a clockwise-rotation circulatory flow. It is the base state, denoted by $\hat{\mathbf{x}}$, on which vibrational forcings are imposed.

The present introduction of vector and base fields, $\mathbf{v}$ and $\hat{\mathbf{x}}$, allows us to apply the optimization theory explained in the previous section. In the theory, however, the way of vibrational forcing is restricted to the case that $M(\hat{\mathbf{x}})=0$. Then the optimal vibrational form $\hat{\mathbf{b}}_{\text {opt }}$ obtained corresponds to the optimal spatial distribution of the following complex vibrational vorticity source $S_{\zeta}$ and heat source $S_{\theta}$, included in the vorticity and temperature equations: 


$$
\begin{aligned}
& \frac{\partial \zeta}{\partial t}+\frac{\partial(u \zeta)}{\partial x}+\frac{\partial(v \zeta)}{\partial y}=G r \frac{\partial \theta}{\partial x}+\Delta \zeta+a e^{i \omega t} S_{\zeta}(x, y)+c . c ., \\
& \frac{\partial \theta}{\partial t}+\frac{\partial(u \theta)}{\partial x}+\frac{\partial(v \theta)}{\partial y}=\frac{1}{\operatorname{Pr}} \Delta \theta+a e^{i \omega t} S_{\theta}(x, y)+c . c .
\end{aligned}
$$

The optimization method makes it possible to have the spatial distribution without any parametric studies.

For a normalization condition, the positive constant $\sigma$ in Eq. (6) must be $\sigma=N^{2}$ so that the sum of spatial integral of absolute value squared of the sources would be constant at unity as the division number $N$ tends to infinity, that is

$$
\lim _{N \rightarrow \infty}\|\hat{\mathbf{b}}\|^{2}=\int_{V}\left(c\left|S_{\zeta}\right|^{2}+\left|S_{\theta}\right|^{2}\right) d V=1,
$$

where $V$ denotes the domain of the square cavity. The choice plays an essential role in convergent properties discussed in the next section. In this study, a dimensionless positive number $c$ is held fixed at unity. It is a special case as the first attempt of optimization. As we shall see, the amplitude of optimal vibrational vorticity source is far smaller than that of heat source, insensitive to the value.

Other reasonable constraints can modify the definition of the norm. Providing it has the form of Eq. (9), including the above norm (12), we can instead solve the eigenvalue problem (10) and obtain a optimal solution depending on the norm. For instance, time-averaged quantity of spatially integrated enstrophy on the domain corresponds to the time-averaged energy input. Based on the first-order approximation, the constraint of fixing the input is of the form (9). Let us confirm herein that the main objective of this study is to present such a general framework of non-parametric, non-iterative optimization on ODEs (1).

\section{Results and Discussions}

Given the vector field $\mathbf{v}$ and a vibrational form, we can confirm the applicability of the approximations and the optimization theory, introduced in the previous section. First of all, the second-order approximation is numerically validated by comparison with a direct computation without any approximations. Such a validation has already been made on a base state with a constant heat-flux on the left-hand side wall $[33,34]$. In this section, it is validated for a base state with a constant temperature on the sidewall, based on the boundary condition of this study. Next, the thermal convections optimizing dimensionless heat transfer rate, Nusselt's number, are presented and, finally, we confirm they are indeed optimal solutions by use of direct computations.

\subsection{Numerical validation of approximated solutions}

Figure 3 shows steady response of streamfunctions to a left-hand sidewall temperature variation, given by $\left.\theta\right|_{x=0}=1+a \sin \omega t$, where $a=0.2, \omega=250$, and division number $N=100$. The flow fields obtained by the second-order approximation are in good agreement with those by the direct computation.

In order for quantitative confirmation, the streamfunctions at the center line $(x=0.5)$ at each phase are shown in Fig. 4. In this figure thermal convections are computed by four methods, i.e. direct computation without approximation, and truncated perturbation expansions up to the third-order. For the case of $a=0.2$, the results of approximations except for the first order are almost identical with those of the direct computation. On the other hand, for a slightly larger amplitude case $(a=0.4)$, we can find that the third-order approximation is better than the second-order one for some phases. In total, however, the second-order approximation well recovers the results of direct computations while the amplitude $a$ is sufficiently small, i.e. $a \leq 0.4$. 
Herein, please note that whether or not the amplitude $a$ is small enough depends on the way of vibration, quantified by $M(\hat{x})$ and vibrational form $\hat{\mathbf{b}}$. The larger the norm of these quantities, the smaller the amplitude $a$ should be. For the case of the present vibration, the norm of $\hat{b}$ is very large, evaluated to be the order of $10^{3}\left(N^{3 / 2}\right)$, diverging as $N$ tends to infinity. Moreover, it is well known that the base thermal convection field of this study has an intrinsic vibrational mode of internal gravity wave (IGW) [44-46], caused by density or temperature gradient in the direction of gravity acceleration. The natural frequency in a rectangular cavity has been obtained by Thorpe [45] for inviscid fluid, estimated at around $\omega=250$ in the present square cavity. Therefore, the amplitude of forced vibrations can be relatively large at this frequency. Taking these issues into consideration, we can expect that the second-order approximation is applicable in many practical situations.

\subsection{Optimization of time-mean Nusselt's number}

We can easily confirm that the (surface-averaged) Nusselt number $N u$ on the left-hand side wall is a function of a phase (state) point $\mathbf{x}$, introduced in Sec. 2.3, of the form $N u(\mathbf{x})=C+\mathbf{d}^{T} \mathbf{x}$ with a constant $C$. Thus the optimization of time-mean value of $N u$, subject to the constraint (6) on the vibrational form $\hat{\mathbf{b}}$, is reduced to the eigenvalue problem (7). The largest and smallest eigenvalues are shown in Fig. 5. The optimized Nusselt number $\overline{\overline{N u}}_{\text {opt }}$ is expressed by the optimal eigenvalue $\lambda_{\text {opt }}$ $\left(\lambda_{\max }\right.$ or $\left.\lambda_{\min }\right)$ as follows

$$
\overline{\overline{N u}}_{o p t}=N u(\hat{\mathbf{x}})+a^{2} \sigma \lambda_{o p t}\left\|\hat{\mathbf{b}}_{\text {opt }}\right\|^{2},
$$

and the optimal vibrational form $\hat{\mathbf{b}}_{\text {opt }}$ is its corresponding eigenvector. If the optimal form converges as $N$ approaches infinity by an appropriate definition of its norm, and also if the optimal Nusselt number converges by the property of the Boussinesq equation system, the value $\sigma \lambda_{\text {opt }}$ also does. That is the reason why the vertical axis in the figure is taken to be $\sigma \lambda$.

Except for some lower-frequency cases where it increases slightly at $N=100$, the optimal eigenvalue $\sigma \lambda_{\text {opt }}$ converges well by $N=80$ and, therefore, these results show the optimal properties of the Boussinesq equation system, independent of the division number $N$.

Figure 6 shows the distribution of optimal vibrational heat source to maximize $\overline{\overline{N u}}$ at $\omega=240$. We can confirm that its amplitude converges sufficiently at $N=60$, and that its phase is also in the process of convergence. They are also regarded as the optimal properties of the Boussinesq equation system.

The dependency of the optimal eigenvalue on the angular frequency $\omega$ is shown in Fig. 7. The minimum eigenvalue is monotone increasing and approaches to zero with $\omega$. In contrast, the maximum value has three local maxima at $\omega=240,360$ and 450, which includes the above-explained natural frequency of IGW.

The frequencies can be explained by the linear stability analysis of the steady solution $\hat{\mathbf{x}}$. Figure 8 shows the result of eigenvalue analysis of $J(\hat{\mathbf{x}})$. Among Hopf modes with complex eigenvalues, the imaginary parts of three eigenvalues that have relatively large real parts agree well with the three angular frequencies. Similar coincidence has been reported in our previous study [33] of the case of total amplitude maximization. At all events, the intrinsic vibrational modes of base state $\hat{\mathbf{x}}$ are reflected in the imaginary parts, and the maxima of Nusselt's number can be caused by the nonlinear effects of the optimal large-amplitude forced oscillation imposed on the intrinsic modes.

At the angular frequency of IGW $(\omega=240)$, the optimal forced oscillation are shown in Fig. 9. The maximized thermal convection inclines isotherms to the right side at a phase while it does not incline isotherms to the left side, keeping on average the thermal boundary layer near sidewalls thin. Conversely, the minimized thermal convection is weak, keeping in total the boundary layer thick.

The vibrational form, i.e. vibrational heat and vorticity sources, to induce such thermal convections are shown in Fig. 10. As the increment, or the amplitude, of the vorticity source is tiny, the above-mentioned thermal convections are dominated by the buoyancy force associated with 
vibrational heat source. The source for maximization has two peaks on right and left halves, and they are antiphase as shown in the distribution of rescaled phase. They are similar to the distribution to resonate with IGW [33], which causes alternative inclinations of isotherms.

In contrast, the effects of two peaks of vibrational heat source for minimization, positioned at upper-left and lower-right corners, seem to be cancelled out by those of other adjacent, antiphase two peaks. That is the reason why the resultant thermal convection is gentle.

\subsection{Application and validation of optimization method}

The optimal vibrational form, i.e. optimal vibrational vorticity and heat sources, provides us with vast pieces of information to realize the optimal vibration as much as possible. For the present physical model, the vibrational heat source is realized by the Peltier device and, fortunately, the vorticity source can be negligible. Referring to the vibrational form $\hat{\mathbf{b}}_{\text {opt }}^{(\max )}$ for maximization (Fig. 10), we consider the following simple, feasible vibrational form $\hat{\mathbf{b}}_{0}$, defined by

$$
\begin{aligned}
& S_{\zeta}=0, \\
& S_{\theta}= \begin{cases}1 /(\sqrt{2} \Delta) ; & 0.05 \leq x \leq 0.05+\Delta, 0.65 \leq y \leq 0.65+\Delta, \\
-1 /(\sqrt{2} \Delta) ; & 0.65 \leq x \leq 0.65+\Delta, 0.05 \leq y \leq 0.05+\Delta, \\
0 ; & \text { elsewhere }\end{cases}
\end{aligned}
$$

where $\Delta=0.3$. As explained in the previous section, the vibrational heat source mimics the optimal one that has two antiphase peaks near upper-left and lower-right corners. The linearly independent, normalized vibrational forms, $\hat{\mathbf{b}}_{o p t}^{(\max )}$ and $\hat{\mathbf{b}}_{0}$, span a two-dimensional vector space. In this section, we consider a normalized vibrational form $\hat{\mathbf{b}}(s)$ of a real variable $s(-1 \leq s \leq 1)$ to connect continuously between the two vectors on the space, defined by

$$
\hat{\mathbf{b}}(s)=\frac{s}{s_{0}} \hat{\mathbf{b}}_{0}+\left(\frac{\alpha}{|\alpha|} \sqrt{1-s^{2}}-\frac{s}{s_{0}} \alpha\right) \hat{\mathbf{b}}_{o p t}^{(\max )},
$$

where

$$
\alpha=\hat{\mathbf{b}}_{o p t}^{(\max ) *} \hat{\mathbf{b}}_{0} \equiv\left(\hat{\mathbf{b}}_{o p t}^{(\max )}, \hat{\mathbf{b}}_{0}\right)
$$

and

$$
s_{0}=\sqrt{1-|\alpha|^{2}}
$$

Herein, the parameter $s$ corresponds to the sine of the angle between the vectors $\hat{\mathbf{b}}$ and $\hat{\mathbf{b}}_{\text {opt }}^{(\max )}$, satisfying

$$
\begin{aligned}
\hat{\mathbf{b}}(0) & =\frac{\alpha}{|\alpha|} \hat{\mathbf{b}}_{o p t}^{(\max )}, \\
\hat{\mathbf{b}}\left(s_{0}\right) & =\hat{\mathbf{b}}_{0} .
\end{aligned}
$$

For the present definition (13) of the form $\hat{\mathbf{b}}_{0}, s_{0}$ is about 0.89 .

Figure 11 shows the variation of the increment $\Delta \overline{\overline{N u}}(\equiv \overline{\overline{N u}}-N u(\hat{\mathbf{x}}))$ of time-mean Nusselt's number for the vibrational form $\hat{\mathbf{b}}(s)$, normalized by $\sigma \lambda_{\max }$. The computations were conducted by the direct method without approximations: substituting the vibrational form (14) into the equation system (11), we can solve directly the equation system by use of the finite difference method. The amplitude $a$ is fixed at unity. 
At $s=0$ the normalized increment reaches a local maximum of about unity (0.98752), indicating that the vibrational form $\hat{\mathbf{b}}_{\text {opt }}^{(\max )}$, obtained in the previous section, indeed maximize the time-mean Nusselt's number. It must be emphasized that the fact sorely relies on the extent of the approximation to the real vibration: as long as the second-order approximation is effective, the optimal vibrational form obtained by the formulation (7) must be globally optimal mathematically. Therefore, the result can be regarded as a further validation of the second-order approximation: minute difference from unity at $s=0$ is originated from third- or higher-order effects with some numerical errors.

In addition, we can find the increment of the Nusselt number accomplished by the feasible vibrational form (13) exceeds $20 \%$ of the optimal value. The eigenvectors of a Hermitian matrix are orthogonal and, therefore, the inner product $\alpha$ of a feasible candidate of vibrational form and the optimal one can be a measure of the extent of optimization [33]. It provides the methodology to select a desirable one among possible candidates.

\section{Conclusions}

This study presents a non-parametric, non-iterative optimization of time-mean value of an inner-product-type index on any non-autonomous ODEs when a base field without forcing is stable steady state and the amplitude $a$ of vibrational forcing is sufficiently small. It is based on a perturbation expansion with the amplitude $a$ taken as a perturbation parameter, and the second-order truncated expansion, i.e. the second-order approximation, provides the method that reduces the optimization problem to an eigenvalue one. Discretization of a partial differential equation system allows us to apply the method to a wide class of dynamical system. As an example, it is applied to optimize time-mean Nusselt's number on a thermal convection field in a square cavity with horizontal temperature difference. The main results are as follows:

1. The second-order approximation well approximates to the real forced oscillation induced by sinusoidal temperature variation on the left-hand side wall at the frequency of internal gravity wave (IGW). The amplitude of forced oscillation is relatively large in this case because the base thermal convection field has an intrinsic vibrational mode at this frequency and, in addition, the norm of vibrational form, i.e. a vector to specify the way of vibrational forcing, is very large. Therefore, the result indicates that the approximation can be useful in many practical situations.

2. The optimal thermal convection to maximize the Nusselt number is similar to the resonant thermal convection of IGW. The vibrational heat source has two peaks on left- and right-hand sides each, in accordance with the property of the vibrational form to induce IGW resonance, reported in our previous study [33]. In contrast, the thermal convection to minimize the Nusselt number is gentle: adjacent, antiphase two peaks of vibrational heat source cancel each other out.

3. As long as the second-order approximation well approximates the real forced vibration, it is certain that the vibrational form obtained by the proposed method globally optimize the time-mean value of a given inner-product type index. We numerically confirmed that the optimal vibrational form obtained actually maximize the time-mean Nusselt number. Moreover, a feasible vibrational form that mimics the optimal one accomplished $20 \%$ of the optimal increase. The inner-product of the optimal and feasible vibrational forms provides a measure to quantify the extent of optimization, allowing us to choose desirable one among possible ways of time-varying forcing.

The optimal solution obtained by the present method makes physical sense while the amplitude $a$ of vibration is sufficiently small and the second-order truncated expansion well approximates the real vibration. However, it is definitely the globally optimal asymptotic solution as $a$ approaches to zero, being an ideal initial guess for applying a nonlinear programming when $a$ is relatively large. The applicability to many situations, including the extension of constraints, is an issue in the future. 


\section{Acknowledgement}

This work was supported by JSPS KAKENHI Grant Number JP16K06157.

\section{References}




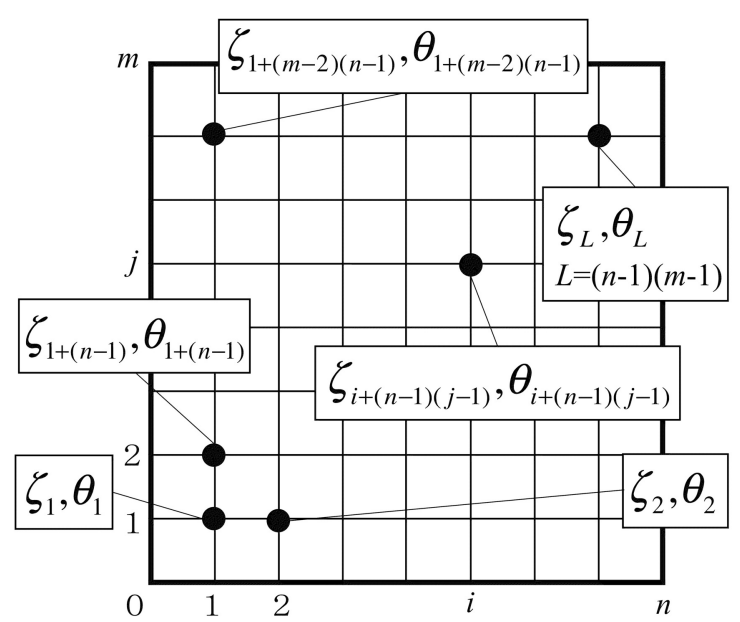

Figure 2. Definition of a phase space and a phase point $\mathbf{x}$. The division number in the $x$ and $y$ directions are $n$ and $m$, respectively. In this study, they are identical with $N$. Both of temperature $\theta$ and vorticity $\zeta$ are defined on each inner lattice point, organizing a phase point $\mathbf{x}$.

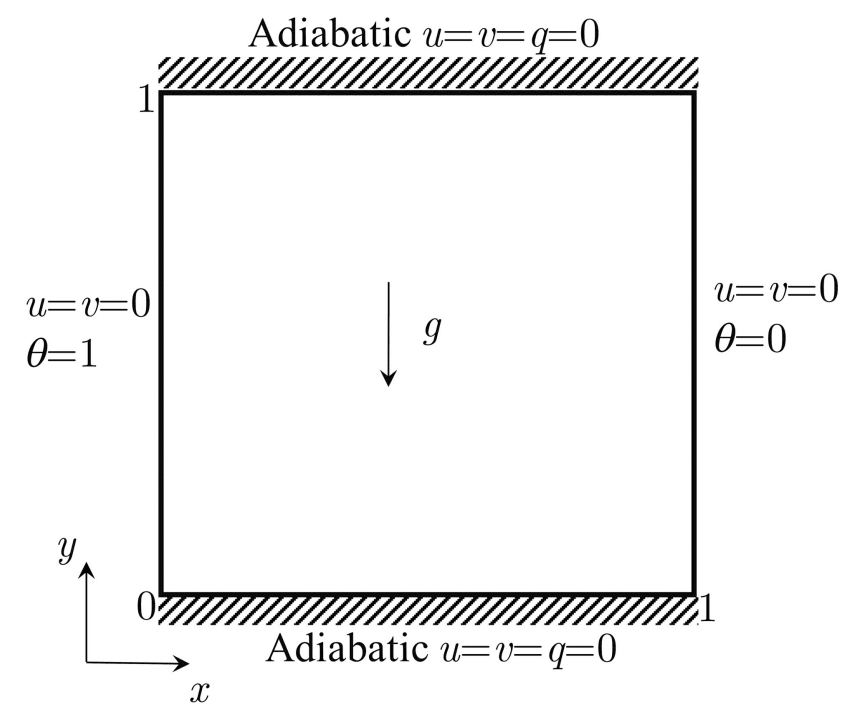

Figure 1. Physical model 

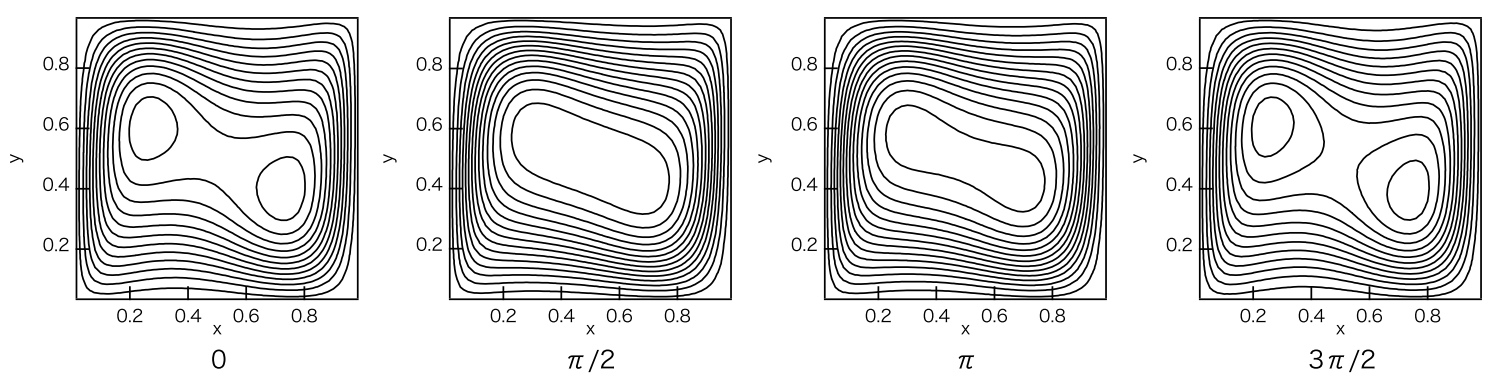

(a) direct computation
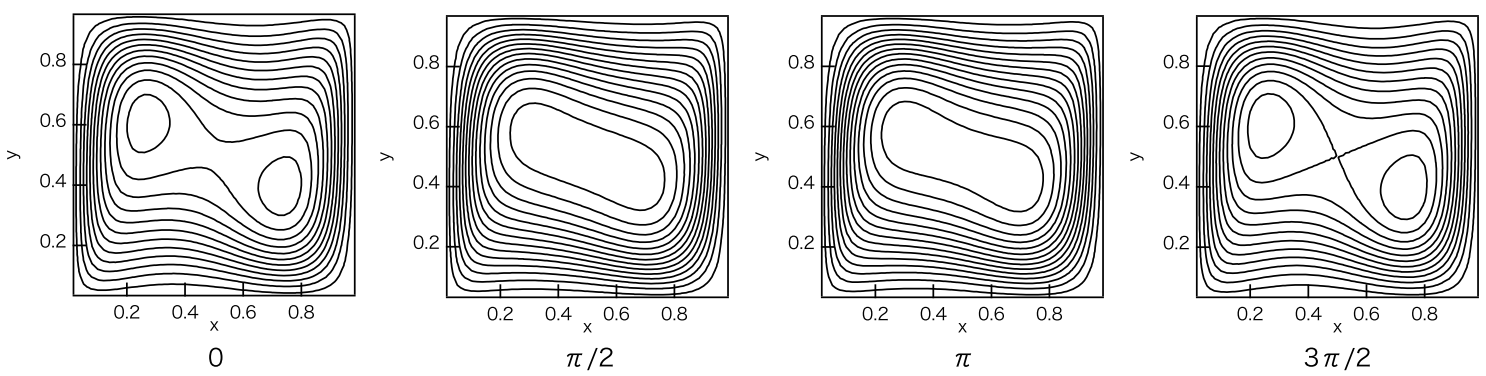

(b) second-order approximation

Figure 3. Streamlines of the steady response at $a=0.2, \omega=250$ and $N=100$, computed by (a) the direct method and (b) the second-order approximation. The increment is 1.0, and outermost is zero. They are drawn for every $\pi / 2$ phase.
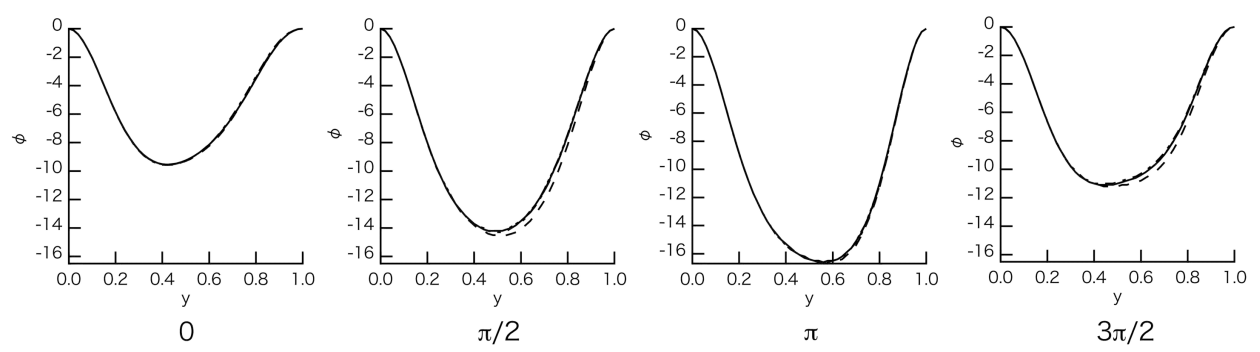

(a) $a=0.2$
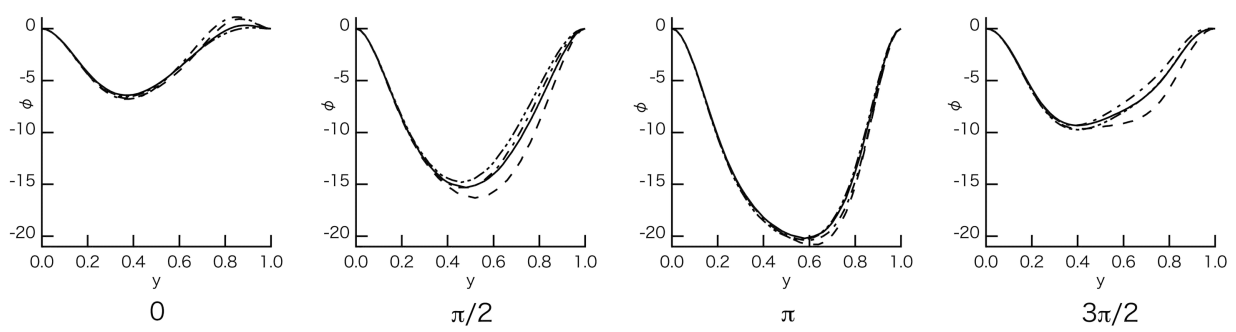

(b) $a=0.4$

Figure 4. Streamfunctions on the midplane $(x=0.5)$ at $\omega=250$ and $N=100$ : (a) $a=0.2$ and (b) $a=0.4$. They are drawn for every $\pi / 2$ phase. Solid, dashed, dash-dotted, and dashed-two dotted lines indicate the results computed by direct method, the first-, second-, and third-order approximations, respectively. For the case of $a=0.2$, it is difficult for us to distinguish with each other except for the result of the first-order approximation. When $a$ is raised up to 0.4 , the difference appears. In total, however, the second-order approximation agrees well with the actual forced vibration. 


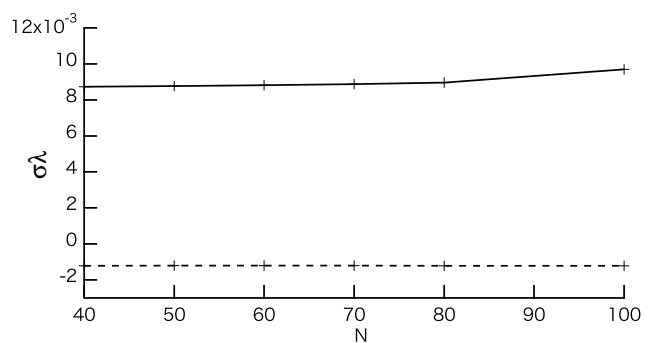

(a)

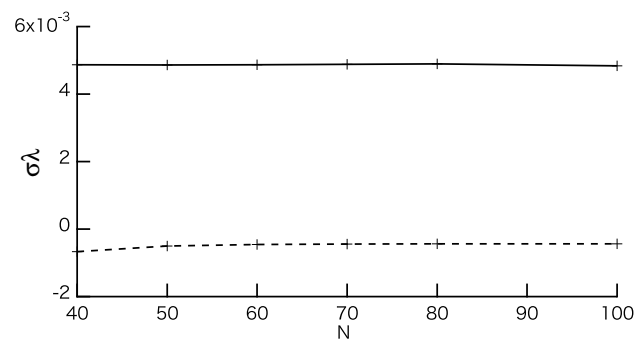

(c)

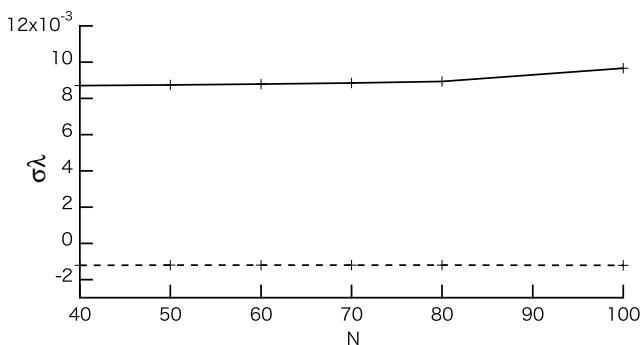

(b)

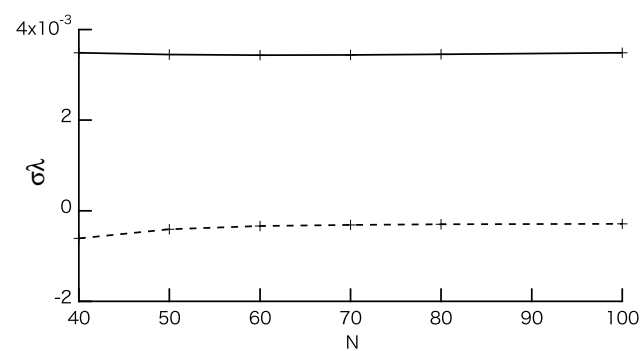

(d)

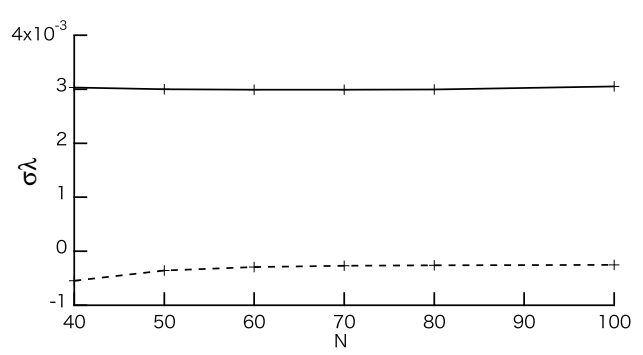

(e)

Figure 5. The variation of $\sigma \lambda$ with division number $N$ : (a) $\omega=0$; (b) $\omega=2$; (c) $\omega=240$; (d) $\omega=360$; (e) $\omega=450$. Solid and dashed lines indicate the maximum and minimum eigenvalues, respectively. All eigenvalues converges well to their limit values by $N=80$. In some cases, the eigenvalue deviates from the value at $N=100$, which is caused by numerical errors. 

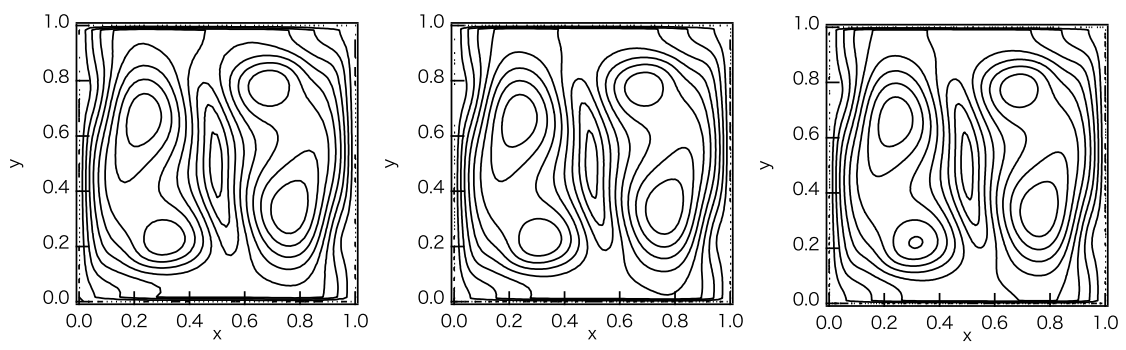

(a) amplitude
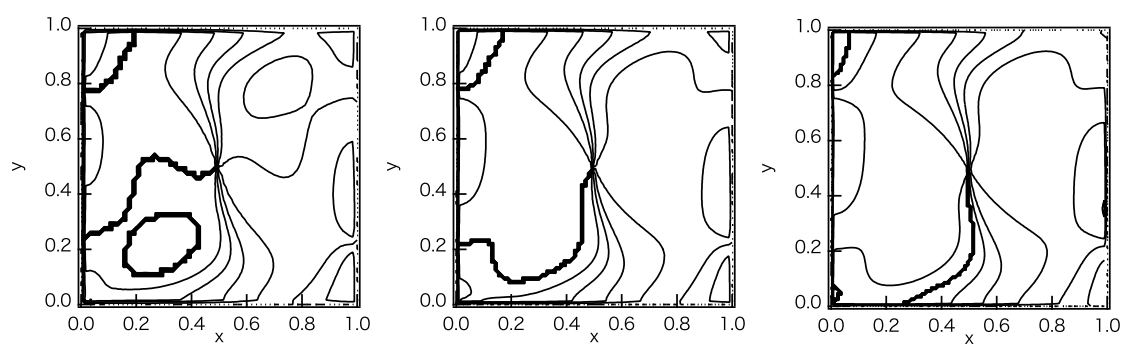

(b) rescaled phase

Figure 6. Vibrational heat source versus division number $N$ for maximizing the Nusselt number at $\omega=240$ : (a) amplitude of the heat source (increment 0.2 ) and (b) rescaled phase of the source (increment 0.2). The left, center and right figures are the case of $N=60,80$ and 100, respectively. The original phase ranges $0 \leq \theta \leq 2 \pi$, rescaled as $-1 \leq \theta^{\prime} \equiv \theta / \pi-1 \leq 1$. In the figures of the rescaled phase, false discontinuity between $\theta^{\prime}=-1$ and 1 is indicated by thick solid line. The amplitude sufficiently converges by $N=60$. Conversely, the phase is in the process of convergence by $N=100$.

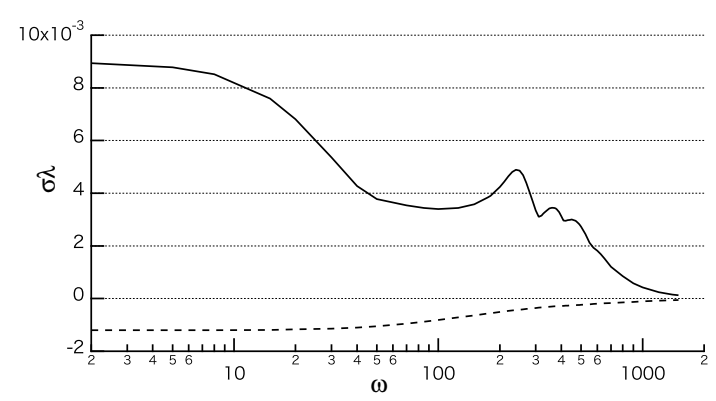

Figure 7. The variation of optimal eigenvalues with the angular frequency $\omega(N=80)$. The solid and dashed lines are the maximum and minimum eigenvalues, respectively. The maximum one has local maxima at $\omega=240,360$ and 450 . The frequency of 240 is in accordance with that of the internal gravity wave (IGW). 


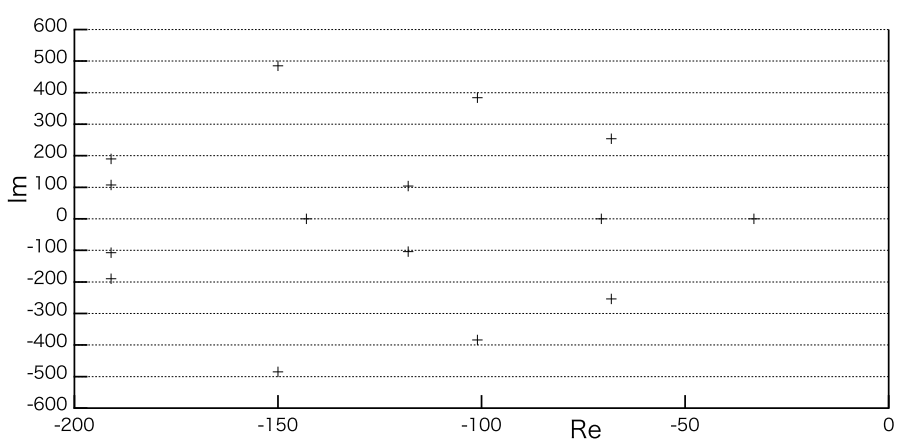

Figure 8. Typical eigenvalues of a Jacobian matrix $J(\hat{x})$. The horizontal and vertical axes indicate the real and imaginary parts of eigenvalues, respectively. 

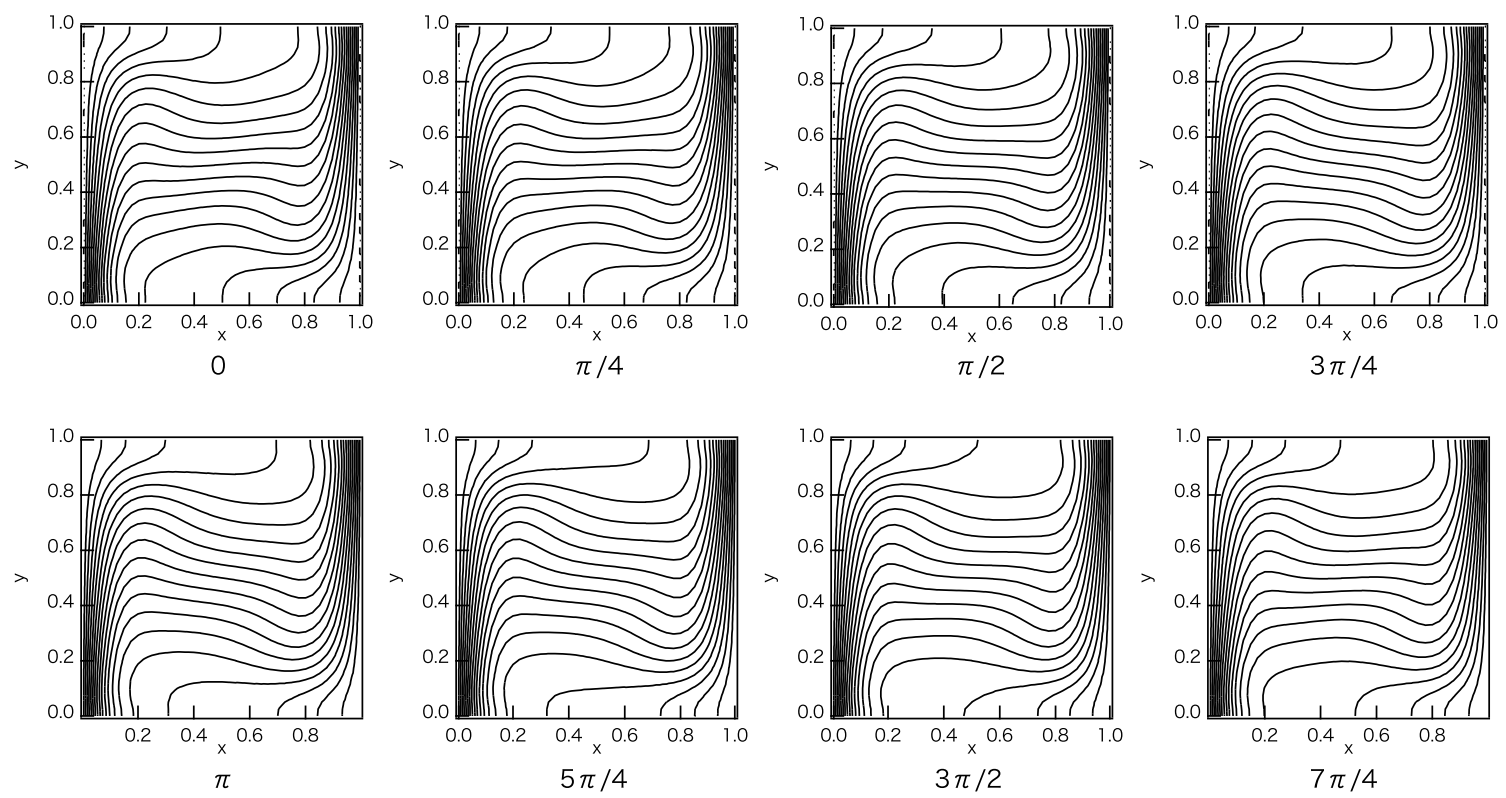

(a) streamfunction for maximization
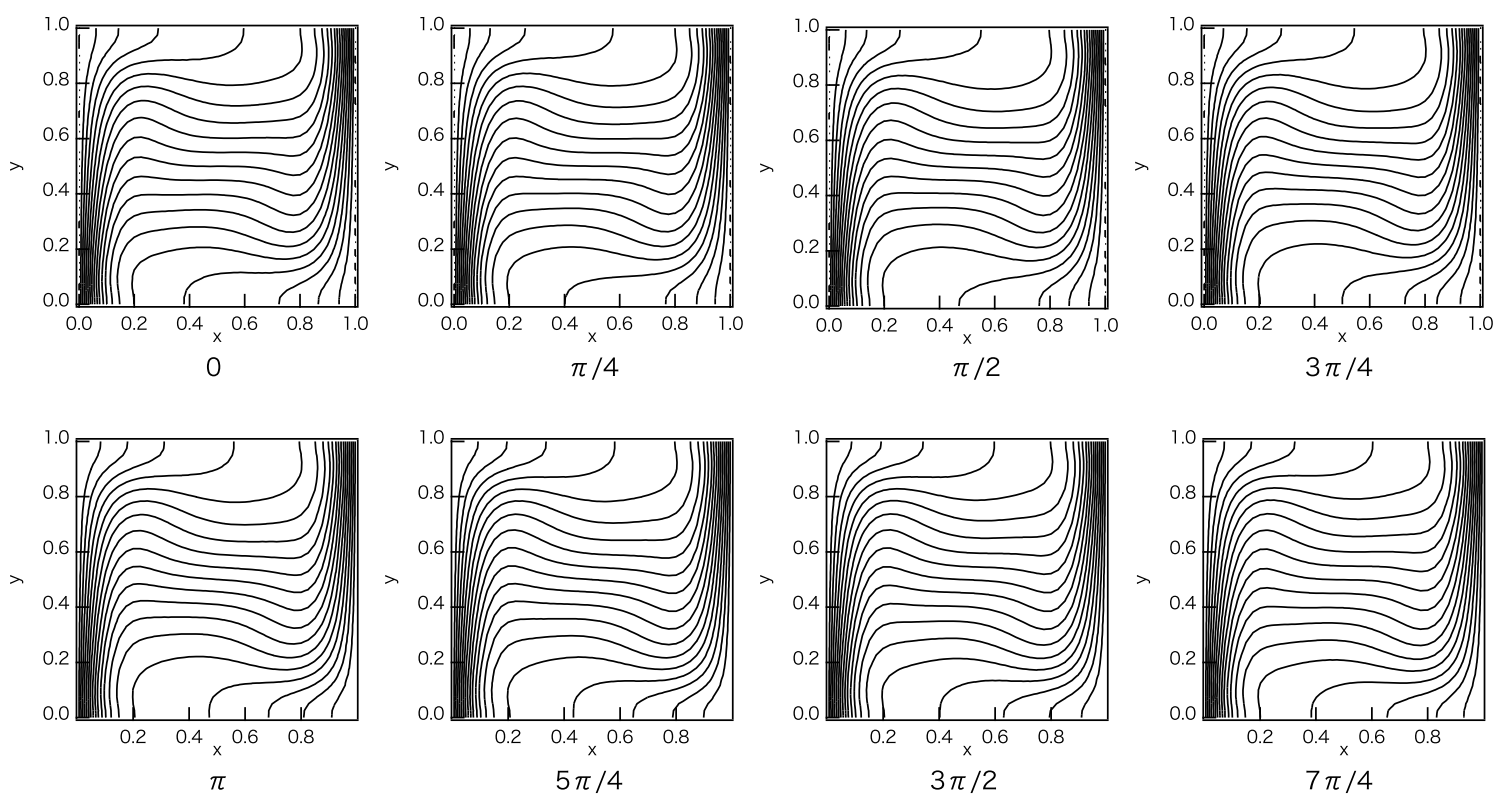

(b) streamfunction for minimization

Figure 9. Steady response to the optimal vibrational form, computed by the second-order approximation at $a=1.0, \omega=240$ and $N=80$ : (a) isotherms for maximizing time-mean Nusselt's number; (b) isotherms for minimization. Outermost is 0 . Increment is 1.0 . They are drawn for every $\pi / 4$ phase. 

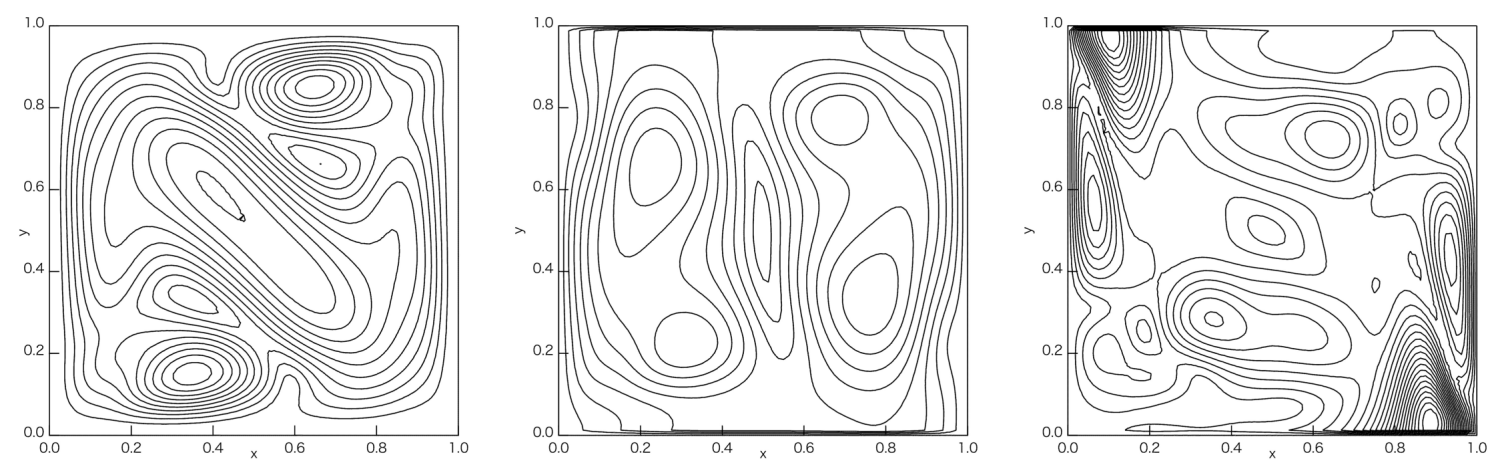

(a) amplitude
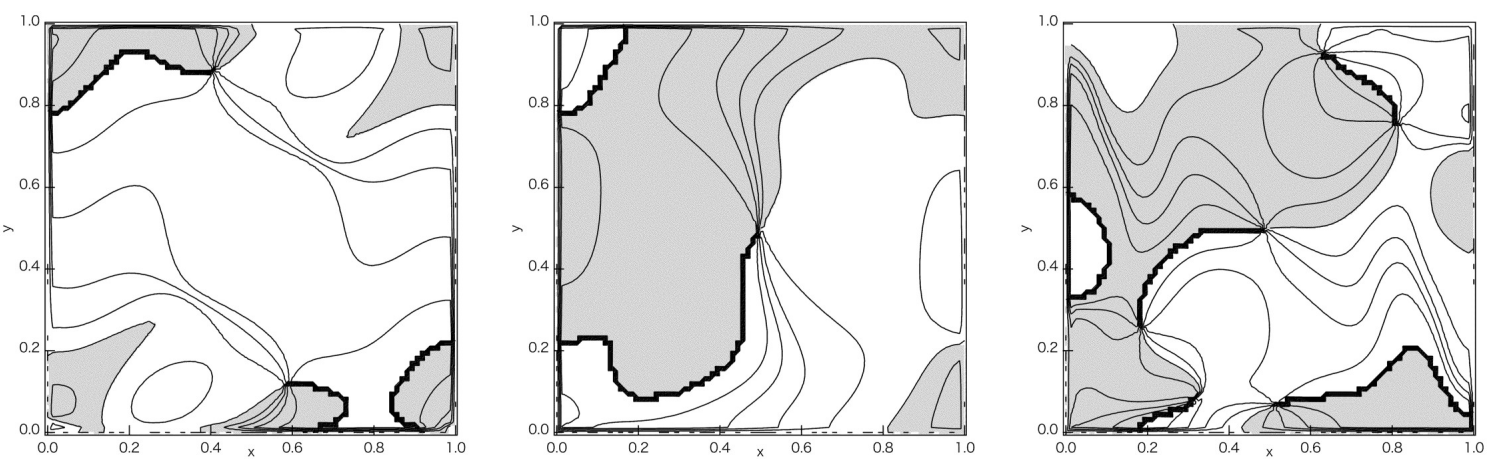

(b) rescaled phase

Figure 10. Optimal vibrational sources at $\omega=240$ and $N=80$ : (a) the amplitude of the vibrational heat and vorticity sources, and (b) the rescaled phase of the sources. The left, center and right figures are vorticity source for maximization, heat source for maximization, and heat source for minimization, respectively. The increment of the amplitude of vorticity and heat sources are $2.0 \times 10^{-5}$ and 0.2 , respectively. Outermost is 0 . The increment for the phase is 0.2 . The phase has a degree of freedom of a constant and, in this study, the phase of vibrational heat source at the center $(x, y)=(0.5,0.5)$ is fixed at zero, which determines the phase of all components of vibrational form including vibrational vorticity source. The shaded region is positive.

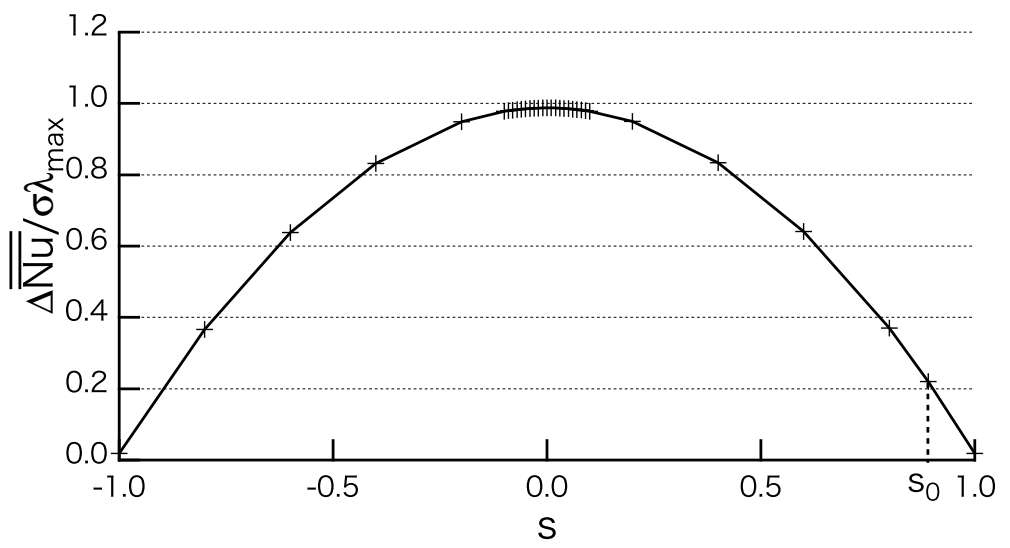

Figure 11. Increase $\Delta \overline{\overline{N u}}$ of time-mean Nusselt's number when vibrational forcings are imposed, normalized by $\sigma \lambda_{\max }$. A variable $s$ denotes the sine of the angle between the optimal vibrational form $\hat{\mathbf{b}}_{\text {opt }}^{(\text {max })}$ for maximizing the time-mean Nusselt's number and a given vibrational form $\hat{\mathbf{b}}(s)$ (Eq. (14)). At $s=s_{0}$, the form $\hat{\mathbf{b}}(s)$ becomes a feasible vibrational form, defined by Eq. (13). 


\section{References}

1. J. C. Hermanson, M. G. Mungal, and P. E. Dimotakis, AIAA J. 25, 578 (1987).

2. A. K. Charakopoulos, T. E. Karakasidis, P. N. Papanicolaou, and A. Liakopoulos, Phys. Rev. E 89, 032913 (2014).

3. H. Sakashita, Int. J. Heat Mass Transfer 93, 1000 (2016).

4. P. Bräunlich, J. Gasiot, J. P. Fillard, and M. Castagne, Appl. Phys. Lett. 39, 769 (1981).

5. M. R. Mross and J. E. Walsh, Phys. Fluids 19, 1217 (1976).

6. T. S. Team, G. Giruzzi, F. Imbeaux, J. L. Ségui, X. Garbet, G. Huysmans, J. F. Artaud, A. Bécoulet, G. T. Hoang, X. Litaudon, et al., Phys. Rev. Lett. 91, 135001 (2003).

7. S. R. Tieszen, T. J. O'hern, E. J. Weckman, and R. W. Schefer, Combust. Flame 139, 126 (2004).

8. P. M. Ligrani, J. L. Harrison, G. I. Mahmmod, and M. L. Hill, Phys. Fluids 13, 3442 (2001).

9. J. Friedrich, Y.-S. Lee, B. Fischer, C. Kupfer, D. Vizman, and G. Müller, Phys. Fluids 11, 853 (1999).

10. M. Shapiro and H. Brenner, Phys. Fluids A 2, 1744 (1990).

11. M. B. Chiekh, M. Ferchichi, J.-C. Béra, and M. Michard, J. Fluids Struct. 54, 522 (2015).

12. Z. Wang and I. Gursul, AIAA J. , 1 (2017).

13. A. P. Baskakov, B. V. Berg, O. K. Vitt, N. F. Filippovsky, V. A. Kirakosyan, J. M. Goldobin, and V. K. Maskaev, Powder Tech. 8, 273 (1973).

14. L. R. Glicksman and A. W. Hunt Jr, Int. J. Heat Mass Transfer 15, 2251 (1972).

15. T. Khan and R. Turton, Int. J. Heat Mass Transfer 35, 3397 (1992).

16. A. Velazquez, J. Arias, and B. Mendez, Int. J. Heat Mass Transfer 51, 2075 (2008).

17. M. Pourgholam, E. Izadpanah, R. Motamedi, and S. E. Habibi, Appl. Thermal Eng. 78, 248 (2015).

18. Z. Cheng, C. Juli, N. B. Wood, R. G. J. Gibbs, and X. Y. Xu, Med. Eng. Phys. 36, 1176 (2014).

19. E. Kidher, Z. Cheng, O. A. Jarral, D. P. O Regan, X. Y. Xu, and T. Athanasiou, J. Card. Surg. 9, 193 (2014).

20. M. A. Stevens, J. Hydr. Engrg. 115, 309 (1989).

21. C. Yundong, L. Xiaoming, W. Erzhi, J. Li, and W. Guang, IEEE Trans. Dielectrics and Electrical Insulation 9, 169 (2002).

22. J. L. Paulsen, J. Franck, V. Demas, and L.-S. Bouchard, IEEE Trans. Magnetics 44, 4582 (2008).

23. Y. Liu, Q. Chen, K. Hu, and J.-H. Hao, Int. J. Heat Mass Transfer 102, 1073 (2016).

24. D. N. Srinath and S. Mittal, Comput. Meth. Appl. Mech. Eng. 199, 1976 (2010).

25. C. Y. Ho and C.-Y. Huang, IEEE Wireless Commun. Lett. 1, 420 (2012).

26. A. Beloglazov and R. Buyya, IEEE Trans. Parallel and Distributed Systems 24, 1366 (2013).

27. P. Venini and M. Pingaro, Struct. Multidisc. Optim. 55, 1559 (2017).

28. M. Hadi and M. R. Pakravan, IEEE Commun. Lett. 22 (2018).

29. L. Fang and X. Li, Appl. Math. Mech. -Engl. Ed. 36, 1329 (2015).

30. I. Grigorenko, M. E. Garcia, and K. H. Bennemann, Phys. Rev. Lett. 89, 233003 (2002).

31. P. A. Nelson, J. K. Hammond, P. Joseph, and S. J. Elliott, J. Acoust. Soc. Am. 87, 963 (1990).

32. X. Chen and J. G. Muga, Phys. Rev. A 82, 053403 (2010).

33. H. Ishida, K. Yamamoto, S. Nishihara, T. Oki, and G. Kawahara, Int. J. Heat Mass Transfer 55, 6618 (2012).

34. H. Ishida, S. Sugimura, T. Kuroda, and G. Kawahara, Int. J. Heat Mass Transfer 96, 145 (2016).

35. G. Z. Gershuni and D. V. Lyubimov, Thermal vibrational convection (John Wiley \& Sons Ltd, 1998).

36. G. Z. Gershuni and Y. M. Zhukhovitskiy, Fluid Mech.-Sov. Res 15, 63 (1986).

37. W. S. Fu and W. J. Shieh, Int. J. Heat Mass Transfer 35, 1695 (1992).

38. H. Ishida and H. Kimoto, Heat Transf.-Asian Res. 29, 545 (2000).

39. G. B. Kim, J. M. Hyun, and H. Sang Kwak, Numer. Heat Transfer A 43, 137 (2003).

40. S. K. Kim, S. Y. Kim, and Y. D. Choi, Phys. Fluids 17, 014103 (2005).

41. E. V. Kalabin, M. V. Kanashina, and P. T. Zubkov, Numer. Heat Transfer A 47, 609 (2005).

42. E. V. Kalabin, M. V. Kanashina, and P. T. Zubkov, Numer. Heat Transfer A 47, 621 (2005).

43. H. Ishida, Y. Takagi, N. Kin, H. Yoshimura, and G. Kawahara, Numer. Heat Transfer A 58, 20 (2010).

44. C.-S. Yih, J. Fluid Mech. 8, 481 (1960).

45. S. A. Thorpe, J. Fluid Mech. 32, 489 (1968).

46. S. Paolucci and D. R. Chenoweth, J. Fluid Mech. 201, 379 (1989) 